\title{
EDITORIAL
}

\section{Dengue in Brazil}

\author{
Dengue no Brasil
}

\section{Luiz Tadeu Moraes Figueiredo ${ }^{1}$}

At this moment, when the Brazilian people feels optimistic about the country development and a promising future, unfortunately it is time to face the reality that dengue, a disease caused by dengue flaviviruses of serotypes 1 through 4 : dengue virus $1,2,3$ and 4 (DENV-1, 2, 3 and 4), remains a national tragedy. Over the last 31 years we have been striken by successive outbreaks of this arboviral disease, transmitted to man by the mosquito Aedes aegypti. The incidence of dengue has been on an upward trend, and in the last decade 700,000 cases have been reported per year. Most Brazilian states are infested by Aedes aegypti and endure dengue transmission. The average age of dengue hemorrhagic fever patients has decreased, turning it into a disease that affects a rising proportion of children. In recent years dengue outbreaks have included many atypical cases, including myocarditis, hepatitis, meningoencephalitis and acute kidney failure, and fatality rates have also increased.

Two papers on dengue, one from North and another from South Brazil, are being published in this issue of the Journal of the Brazilian Society of Tropical Medicine. One paper reports on the dengue situation in 2011 in Manaus, a large city of North Brazil, capital of the State of Amazonas. Patients with acute febrile illness were tested for dengue by reverse transcription polymerase chain reaction (RT-PCR) and nested PCR, and 137 of 432 were found to be positive. Among these, 51 had DENV-2, 31 had DENV-4, 22 had DENV-3 and 17 had DENV-1. Co-infections by more than one DENV serotype were also observed: DENV-4 and -3 in 6 cases, DENV- 1 and -4 in 5 cases, DENV- 1 and -2 in 3 cases, and DENV- 2 and -3 in 2 cases. This is the first report on the simultaneous circulation of the four dengue serotypes in the same place in Brazil, and provides clear evidence of dengue hyperendemicity, a situation that will probably lead to increasing morbidity, severe forms of disease, and mortality.

The second paper reports a study on molecular epidemiology of DENV isolates in the State of Parana, South Brazil, a subtropical region less infested by Aedes aegypti, where dengue outbreaks are less frequent. Ribonucleic acid (RNA) extracts of virus isolates from 11 counties were tested by RT-PCR and nested PCR, and sequences in the genomic region of capsid/premembrane junction of DENV1 and 2 were analyzed. Four patients were co-infected with both

1. Centro de Pesquisa em Virologia, Faculdade de Medicina de Ribeirão Preto, Universidade de São Paulo. Ribeirão Preto, SP.

Address to: Dr. Luiz Tadeu Moraes Figueiredo. Diretor do Centro de Pesquisa de Virologia/FMRP/USP. Av. Bandeirantes 3900, 14049-900 Ribeirão Preto, SP, Brasil. Phone 55 163602-3271

e-mail: 1tmfigue@fmrp.usp.br

Received in 16/05/2012

Accepted in 17/05/2012
DENV-1 and 2. DENV-1 sequences in Parana were 95-100\% similar to those of other DENV-1 Brazilian strains belonging to genotype $\mathrm{V}$, the only genotype circulating in Brazil thus far. The replacements of methionine by alanine (amino acid 69) and isoleucine by valine (amino acid 117), observed in these sequences had been previously detected in DENV-1 strains isolated in Rio de Janeiro and São Paulo, the likely origin of this virus. Similarly, DENV-2 sequences were 98-100\% similar to those of other DENV-2 strains of genotype I (American/Asian). That genotype of DENV-2, which has been associated with dengue hemorrhagic fever, has predominated in Brazil during the 19 years of its circulation (1991 - 2008).

Thus, both papers presently published call attention to the hyperendemicity of DENV in Brazil, with increased genetic diversity and serious consequences, such as an expanded range of pathogenic properties, increased transmissibility and virulence. How did we reach this level of dengue endemicity? How to control dengue in Brazil? The most important tool for dengue prevention so far is vector control. However, we have not been successful in the fight against the mosquito vector. Despite efforts to control Aedes aegypti, a hyper-endemic situation has remained in Brazil, with large outbreaks involving all four dengue serotypes (DENV-1 to 4). Therefore, it is regrettable that an expectation of short term reduction in dengue incidence has been raised by some public health authorities, and by the press, attributed to a triumph of fight against the vector. At this point, a dengue vaccine, which will probably become available in the next few years, remains as the great hope to control this disease in the Brazilian population.

Last but not least, other arboviruses such as yellow fever, Saint Louis encephalitis, oropouche and mayaro are also causing outbreaks and sporadic cases of febrile disease in Brazil. Infections by these agents are commonly confused with dengue and frequently remain misdiagnosed.

\section{CONFLICT OF INTEREST}

The author declare that there is no conflict of interest.

\section{FINANCIAL SUPPORT}

The author receives a Researcher Fellowship from Conselho Nacional de Desenvolvimento Científico e Tecnológico (CNPq).

\section{REFERENCES}

1. Figueiredo LTM. Dengue in Brazil: 1999-2009. Dengue Bullettin (World Health Organization) 2010; 34:6-12. 